\title{
Experiencing Derivations
}

\author{
Tanya Reinhart \\ Tel Aviv University \\ University of Utrecht
}

\section{The problem}

Experiencing verbs allow different syntactic realizations: The experiencer argument can surface as the object, as in (1a), or as the subject, as in (1b). In English, this alternation is restricted to just a few verbs (while the others use various passive forms for (1b)). But in many languages it is fully productive. In Hebrew, e.g. all object-experiencing verbs have also a subject-experiencing alternate, as in (2), but the verb's morphology differs in the two alternates.

(1) a. Something worries Lucie.

\section{experiencer}

b. Lucie worries (about something)

experiencer

(2) a. ha-olam hid'ig / hirgiz/ rigesh/ hivhil et Max.

The world worried/ angered / excited / scared (acc) Max.

b. Max da'ag / hitragez / hitragesh /nivhal (la/al/me ha-olam)

Max worried /angered / excited (prep. the world)

Assuming that the thematic structure of the two derivations is identical, this poses a problem to any approach to the mapping (linking) from lexicon to syntax. Belletti and Rizzi (1988) argued that the two derivations have, in fact, the same underlying structure - the one in (3), where both arguments originate internally. If the theme arguments moves to IP, we obtain (1a), and if the experiencer moves, (1b) is derived. Under this analysis, then, experiencing verbs are unaccusative (since both arguments originate internally). ${ }^{1}$

$$
\begin{aligned}
& \text { DS of both (1a) and (1b): [IP e [vp [worry something] Lucie] ] } \\
& \text { theme experiencer }
\end{aligned}
$$

The most impressive argument of Belletti and Rizzi for this analysis was the anaphora patterns of object-experiencing derivations, e.g. that bound anaphora is permitted in $(4 a, b)$. (As has been observed before, there is a clear contrast between these sentences and, say, (4c), which is a standard weakcrossover violation.)

(4) a. $\left[\mathrm{His}_{\mathrm{i}} \text { health }\right]_{\mathrm{j}}$ worries $\mathrm{e}_{\mathrm{j}}$ every patient $\mathrm{i}_{\mathrm{i}}$

R. Hastings, B. Jackson and Z. Zvolenszky (eds), SALT XI 365-387, Ithaca, NY: Cornell University. 
b. $\quad\left[\mathrm{His}_{\mathrm{i}} \text { solution }\right]_{\mathrm{j}}$ appealed $\mathrm{e}_{\mathrm{j}}$ to every student $\mathrm{i}_{\mathrm{i}}$

c. $\quad *\left[\mathrm{His}_{\mathrm{i}}\right.$ doctor $_{\mathrm{j}}$ visited every patient $\mathrm{i}_{\mathrm{i}}$

This anaphora pattern follows, since in their system the subject originates as the internal theme argument, and in its original position (illustrated in (3)), it is ccommanded by the experiencer, which is higher in that VP.

However, Pesetsky (1995) has shown that there exist, in fact, two classes of experiencing verbs, with very different syntactic properties: The large worry (preoccupare) set does not show any unaccusative properties. A smaller set of the appeal (piacere) type does show indeed unaccusative syntax. Belletti and Rizzi conflated these two types, and a careful examination shows that their analysis is relevant only for the appeal set. Let us verify this difference with just one of the diagnostics used by Pesetsky: Unaccusative verbs do not allow passive, but the worry type verbs do allow it, as in (5).

preoccupare (worry) type:

a. The news worried / surprised /excited Max.

b. Max was worried/ surprised /excited by the news.

piacere (appeal) type:

a. The solution appeals to me /escapes me.

b. $\quad *$ I am appealed by /escaped by the solution

The piacere verbs, by contrast, confirm this unaccusativity diagnostics and disallow passive, as in (6).

Next, Pesetsky shows that it is not the case that the two derivations of the worry type verbs in (1), illustrated again in (7), have identical thematic structure. While both include an experiencer argument, they differ in their second argument. In (7a) the doctor bears the cause role. In (7b), it is the role Pesetsky discovered and labeled subject matter.
a. The doctor worried Lucie. cause experiencer
b. Lucie worried about the doctor. (7a does not entail 7b.) experiencer subject-matter.

To show that the sentences are not thematically identical, Pesetsky points out that they differ in their truth conditions. (7a) does not entail (7b): (7a) can be true if the doctor made Lucie worry about something else, say her health. But (7b) may be false in this situation.

In conclusion, then, there is no basis to assume an unaccusative analysis, along the lines of (3), for the worry type verbs. For all we saw, the derivations in (7) are independent, and the arguments can merge directly to their final positions (with the subject, in both, originating as the external argument). ${ }^{2}$

But this, then, leaves us again with the anaphora problem in (4), repeated.

(4) $\quad\left[\mathrm{His}_{\mathrm{i}} \text { health }\right]_{\mathrm{j}}$ worries $\mathrm{e}_{\mathrm{j}}$ every patient $\mathrm{i}_{\mathrm{i}}$. 
b. [Hisi solution $]_{j}$ appealed $\mathrm{e}_{\mathrm{j}}$ to every student $\mathrm{i}_{\mathrm{i}}$.

We see that regarding anaphora, there is no difference between the two types of verbs: The worry and appeal derivations equally allow it. For the unaccusative appeal case, we may still assume a derivation along the lines represented in (4b) (that can be easily restated within contemporary views on the structure of VP). So the anaphora facts still follow. But the worry type emerges now as a mystery, as we have just dismissed the syntactic analysis represented for it in (4a).

This anaphora puzzle would be my leading question here. But along the road we have to get more specific about the different derivations of the worry type experiencing verbs. As a starting point, we may note that even though the two syntactic realizations of these verbs (e.g.in (1-2) and (7)) are derivationally independent, it is still the same verb in both. So the question still arises what enables the same verb to have such different realizations.

Answering these questions requires a digression into the general framework I am assuming.

\section{Background: The $\theta$-system}

The analysis is based on the $\theta$-system proposed in Reinhart (2000). I survey here only the properties of the system relevant for the present discussion. A more detailed synopsis can be found in Reinhart (2001).

\subsection{Theta features}

Two binary features: +/-c (=Cause change) and +/-m (=Mental state) define eight feature clusters which correspond to what has been labeled $\theta$-roles. The correspondence is not one to one. Some of the feature clusters have varying contextual interpretation (with some of the uses not captured by existing $\theta$-role labels). However, for convenience of reference, I label them in the list below by the role that they are most typically related to.
a. $\quad[+\mathrm{c}+\mathrm{m}]-$
agent
b. $\quad[+\mathrm{c}-\mathrm{m}]-$
instrument
c. $[-\mathrm{c}+\mathrm{m}]-$
experiencer
d. $[-\mathrm{c}-\mathrm{m}]-$
theme / patient
e. $[+c]-$
cause (Unspecified for $/ \mathrm{m}$ ); consistent with either
f. $\quad[+m]-$
(a) or (b).
$?^{3}$
g. $[-\mathrm{m}]-$
(Unspecified for /c): subject matter /locative source
h. $[-c]-$
(Unspecified for $/ \mathrm{m}$ ): Internal roles like goal, benefactor typically dative (or PP).

The clusters (8a-d) are fully specified, with a value for both features. The underspecified features in (8e-h), which I will refer to as unary clusters, have greater interpretative freedom. A verb selecting a $[+c]$ (cause) cluster can realize 
also with an agent or instrument interpretation of this argument (since they are both consistent with $/+\mathrm{c})^{4}$. The unary [-] clusters $(8 \mathrm{~g}-\mathrm{h})$ have the widest range of thematic realizations. They always merge as internal arguments (as we shall see), and they require a preposition (or the dative case) for their thematic specification. The syntactic correlate is that a DP realizing such clusters cannot check accusative case.

\section{2. $[+c]$ subjects}

A class of verbs important for the present discussion is of two (or more)-place verbs with a $[+c]$ cluster (which always realizes externally, as we shall see.) As mentioned, the characteristic property (and hence the diagnostics) of such verbs is that they allow this argument to realize as either an unspecified cause, or as an agent or instrument, as in (9) and (10) .

These verbs are further distinguished by their internal role. In the set (9), it is a theme $([-\mathrm{c}-\mathrm{m}])$.

$$
\begin{aligned}
& V([+c],[-c-m])-\text { break, open... } \\
& \text { a. The wind /Max /the key opened the door } \\
& \text { b. The storm /Max/the stone broke the window. } \\
& \text { c. The earthquake/ Max/ the stick rolled the stone. }
\end{aligned}
$$

In Reinhart (2000, 2001) I argue that universally, all verbs with this feature cluster have an unaccusative alternate, as in The window broke. The converse also holds: All one-place unaccusative verbs have an active alternate with a [+c] role in one language or another.

The set of $[+c]$ verbs which concerns us here is that with an experiencer $([-\mathrm{c}+\mathrm{m}])$ as their second argument, as in (10). These are the worry type verbs in their Object -experiencer realization.

$V([+c],[-c+m] \ldots)-$ worry, amuse, scare, surprise...

a. $\quad$ Max / the noise / the gun worried Lucie.

b. Fred/ Fred's behavior /the discussion surprised Lucie

Fred/ Fred's gedrag /de discussie verbaasde Lucie.

All worry type verbs show this varying interpretation of their external role, and, as we just saw in section 1, they also may have, universally, an alternate without the $[+\mathrm{c}]$ role (Lucie worried (about Max)).

The appeal type verbs never realize with an argument that assumes a cause interpretation. This means that they do not have a $[+c]$ cluster in their basic lexical entry. Although I will not discuss them here, the system entails that both their arguments must merge internally.

\subsection{Reduction}

Various lexicon operations may apply at the verb entry. For the cases under consideration here, I assume that the verb entries in (9) and (10) (with a [+c] cluster) are the basic entries listed in the lexicon, and that their unaccusative and subject-experiencing alternates are derived from them by a reduction operation. 
(11) Reduction of an external $[ \pm \mathrm{c}]$ role -Expletivization.

a. $\quad \mathrm{V}_{\mathrm{acc}}\left(\theta_{1[+\mathrm{c}]}, \theta_{2} \ldots\right)--->\underline{\mathrm{R}}_{\mathrm{e}}(\mathrm{V})\left(\theta_{2} \ldots\right)$

b. $\quad \underline{R}(\mathrm{~V})\left(\theta_{2}\right)<-->\mathrm{V}\left(\theta_{2}\right)$

External role reduction differs from the more familiar internal (reflexivization) reduction in its semantics. The argument reduced by reflexivization is still present in the interpretation (as identical to the surviving argument). But external reduction (expletivization) eliminates the argument altogether. $\mathrm{R}_{\mathrm{e}}$ can be viewed as a semantically null function, as in (15b). The reduced entry denotes just the property corresponding to a one place verb with the remaining argument.

I assume that the ACCusative feature of the verb is assigned already in the lexicon (by a marking procedure I turn to directly). A crucial property of reduction is that it eliminates this feature. (A parametric variation, discussed in Reinhart (2001), is whether the elimination is full or partial, which effects auxiliary selection.) Let us state this in (12) for future reference.

Reduction eliminates the accusative feature of the verb.

External reduction applies in precisely the same way to all $[+c]$ arguments (with no known restrictions). Thus, two of its outputs are illustrated in (13).

$$
\begin{aligned}
& \text { a. } \text { open }_{\mathrm{acc}}\left([+\mathrm{c}],[-\mathrm{c}-\mathrm{m}]--->\underline{\mathrm{R}}_{\underline{\mathrm{e}}} \underline{(0 \mathrm{pen})}[-\mathrm{c}-\mathrm{m}]\right. \\
& \text { b. } \text { worry }_{\mathrm{acc}}\left([+\mathrm{c}],[-\mathrm{c}+\mathrm{m}]--->\underline{\mathrm{R}}_{\underline{\mathrm{e}}}(\text { worry })[-\mathrm{c}+\mathrm{m}]\right.
\end{aligned}
$$

\section{$\underline{R_{e}}($ ppen $)[-c-m]$}

The door $r_{i}$ opened $t_{i}$.

\section{$\underline{\mathrm{R}_{e}}($ worry $)[-\mathrm{c}+\mathrm{m}]$}

Max worried.

But these two reduced entries have dramatically different syntactic realizations: (13a) forces an unaccusative derivation, as in (14), while the derivation involving the reduced experiencing verb in (15) is unergative. (It has been claimed, e.g. by Pesetsky (1995), that (15) too is unaccusative, namely its subject originates internally. But in Reinhart (2000), I argue in some detail that this cannot be the case.)

This difference brings us to the issue of mapping (or linking) from the information in the lexicon to syntactic derivations - the order of merging of the verb's arguments. This is generally faced with thematic hierarchies, or more complex linking rules. Let me summarize how it is captured in the present system. (The mapping view I outline below departs quite radically from my earlier assumptions in Reinhart (2000). It is presented with more detail in Reinhart (2001).)

\subsection{The mapping/linking System}

I follow the notation of Williams (1981), where the mapping instructions are 
built into the lexical entry by indices ( 1 marks an external role, 2 an internal one). Obviously, however, this should not be idiosyncratic information listed individually for each verb. Rather, I assume marking procedures which apply uniformly to all verbs, assigning an index to its arguments (feature clusters). Another information which needs be coded already in the lexicon is the accusative feature of the verb. I argue that whether the verb carries this casefeature is determined by its feature clusters. Hence, this should also be handled in a systematic way by the marking procedures.

Below I list the full set of procedures and other generalizations I am assuming for the mapping problem. Not all of them are crucial for the present discussion, or can be defended and motivated here.

\section{Notation:}

\begin{tabular}{|c|c|c|}
\hline$[\alpha]$ & $=$ & Feature cluster $\alpha$ \\
\hline$/ \alpha$ & $=$ & $\begin{array}{l}\text { Feature (and value) } \alpha . \text { (E.g. the feature } /+m \text { occurs in the clusters } \\
{[+c+m],[-c+m] \text { and }[+m] \text { ) }}\end{array}$ \\
\hline$[/ \alpha]$ & $=$ & $\begin{array}{l}\text { A cluster one of whose features is } / \alpha .(\text { E.g. }[/-c] \text { clusters are [- } \\
\mathrm{c}+\mathrm{m}],[-\mathrm{c}-\mathrm{m}] \text { and }[-\mathrm{c}] .)\end{array}$ \\
\hline [+] & $=$ & $\begin{array}{l}\text { A cluster ALL of whose features have the value +. (E.g. }[-] \\
\text { clusters are }[-\mathrm{c}-\mathrm{m}],[-\mathrm{c}],[-\mathrm{m}] .)\end{array}$ \\
\hline
\end{tabular}

(16) Lexicon marking

Given an n-place verb-entry, $n>1$,

a. $\quad$ Mark a [-] cluster with index 2.

b. Mark a $[+]$ cluster with index 1 .

c. If the entry includes both a $[+]$ cluster a fully specified cluster $[/ \alpha, /-c]$, mark the verb with the ACC feature.

(17) Relevant generalizations of lexical operations:

a. Reduction applies to the marked entry (i.e. after marking).

b. $\quad(=12)$ Reduction eliminates the accusative feature of verb.

(18) CS merging instructions.

a. When nothing rules this out, merge externally.

b. An argument realizing a cluster marked 2 merges internally; An argument with a cluster marked 1 merges externally.

The various thematic hierarchies which have been proposed attempt to define a ranking (hierarchy) for all theta arguments, a project which has encountered many difficulties. The basic idea here is that there are feature-clusters which obligatorily merge externally or internally only, but also others which are not marked, hence they are free to merge in either position, depending on availability of a position, or other CS requirements (such as the accusative case).

The feature clusters fall into the three classes in (19).

$$
\begin{array}{ll}
{[-] \text { clusters: }} & {[-\mathrm{c}-\mathrm{m}](\text { theme })} \\
& {[-\mathrm{c}](\text { goal,..) }} \\
& {[-\mathrm{m}](\text { subject matter,...) }}
\end{array}
$$




\section{[+] clusters: \\ EXPERIENCING DERIVATIONS \\ $[+\mathrm{c}+\mathrm{m}]($ agent $)$ \\ [+c] (cause) \\ $[+\mathrm{m}](?)$ \\ 'mixed' clusters: $\quad[-\mathrm{c}+\mathrm{m}]$ (experiencer) \\ [+c-m] (instrument)}

By (16a), the minus ([-]) clusters are marked 2. Hence, they must merge internally. The plus $([+])$ clusters are marked 1 (by (16b)), i.e. they are obligatorily external. The marking system does not impose any further merging order among the internal [-] roles (which are all marked here with the same index 2, regardless of how many of them the verb has). However, other considerations of the CS may do so: Recall that in the present system, the unary $[-]$ clusters ([-c] and [-m]) require inherent case: Preposition or Dative. Hence, they cannot check the accusative case. This means that there is normally just one argument which can check this case, and this may dictate its merging position.

However, there are two feature clusters in (19) which have 'mixed' values (-are neither [-], nor $[+]$ ), namely those corresponding to experiencer and instrument. Hence, they are not assigned any index by (16). This means that these roles may have varying syntactic realizations. In such cases, (18a) determines that if nothing prevents this (e.g. there is no other argument marked 1 ), these arguments merge externally. (18a could be viewed as some sort of an economy requirement: An external argument is required to check the EPP, if this requirement can be directly met, it is less economical to first merge the argument internally and then move it.)

The ACC-marking procedure in (16c) requires the presence of both a plus cluster $[+]$ and a $[/ \alpha /-c]$ cluster. This captures two conditions on ACC assignment:

a. The ACC feature can be assigned only by a fully specified cluster (as the unary [-] clusters require inherent case). The cluster must, further, contain the feature /-c. This means that only [-c-m] -theme and [-c+m]-experiencer can introduce the ACC feature. (For ECM structures I assume that the complement IP itself realizes this cluster and, thus introduces the ACC feature. In the given system, nothing forces that it must be the same argument which assigns ACC in the lexicon and which checks it in the syntax.)

b. ACC assignment applies only if there is also a [+] cluster in the entry. Recall that a [+] cluster always realizes externally. This condition, then, (combined with (17b)), captures Burzio's generalization that if a verb does not assign an external role, it does not assign an accusative case. Thus, appeal - type verbs which do not have any [+] role in their base entry, cannot have the accusative case, regardless of what the composition of their internal arguments is.

For some illustration of the system, let us check the derivation of (one-place) unaccusative verbs, with the verb drown.

(20) a. Base entry: $\operatorname{drown}([+\mathrm{c}],[-\mathrm{c}-\mathrm{m}])$ 
b. marking: drown $n_{\text {acc }}\left([+\mathrm{c}]_{1},[-\mathrm{c}-\mathrm{m}]_{2}\right.$

c. Reduction: $\mathrm{R}_{\mathrm{e}}(\underline{\text { drown }})\left([-\mathrm{c}-\mathrm{m}]_{2}\right)$

d. $\quad$ Merging: Internal, by (18b): Maxi drowned $t_{i}$

The basic entry is (20a), with both a [+] and a [-] arguments. The marking procedures in (16) apply, as in (20b). If nothing else happens, the entry can be used for a syntactic derivation such as Felix/ the explosion drowned Max. However, expletive reduction can apply to the $[+\mathrm{c}]$ argument, yielding (20c). Reduction eliminates the ACC feature (see 17b), but it has no effects on the remaining arguments. Hence, the theme [-c-m] role is still marked 2. This is an obligatory instruction to merge this verb internally (by 18b). So this is the only option, even though this argument may have to move later to satisfy the EPP.

A well known mapping puzzle is that there are many one place unergative verbs which select a theme argument, and are, thus, indistinguishable thematically from unaccusative verbs (glow shine $_{2}$ buzz $_{2}$ stink $k_{2}$ shudder sweat...). (If unaccusative verbs are listed as such in the lexicon, explaining the different derivations of the two verb-types is a serious problem.) Let us follow the mapping of such unergative entries in the present system.
a. Base entry: glow ([-c-m])
b. Marking: inapplicable (one place entry)
c. Merging: External by (18a). E.g. The diamond glowed.

As defined, the marking procedures in (16) apply to n-place verb entries, such that $\mathrm{n}$ is greater than 1 . Namely, they apply only to entries with at least two arguments. This means that the basic entry illustrated in (34a) remains unchanged and unmarked. Although the argument of glow is a [-] cluster, its merging status ends up here precisely the same as the in other cases where the argument is not marked: (18a) determines that since nothing prevents merging the argument externally, this is the only option. More generally, in the present system, one place verbs are always unergative.

\section{Basic experiencing derivations}

Let us return now to the basic derivations involving experiencing verbs, such as (1), repeated in (21).
a. Something worries Lucie.
cause experiencer
b. Lucie worries (about something)
experiencer subject matter

As we saw in section (1), following Pesetsky 1995, the thematic roles in the object-experiencer and subject-experience derivations are not identical, so the various realizations of the verb allow together three thematic roles: cause: $[+c]$, experiencer: $[-c+m]$ and subject matter, which as I argue directly, corresponds to [-m]. In the present system, this means that all three roles are specified in the basic verb entry, though they need not (in fact, cannot) be all realized in one 
derivation. (I return directly to the issue of their realization options.) As one of the roles is $[+\mathrm{c}]$, reduction can apply to derive a two place verb out of the basic three-place one.

It is convenient to illustrate the relevant derivations with an example from Hebrew, since the basic and the reduced entries have a different morphology.
a. $\quad$ Entry: hirgiz (anger) $([+\mathrm{c}],[-\mathrm{c}+\mathrm{m}] \quad([-\mathrm{m}]))$
(cause, experiencer, subject matter)

b. Marking: hirgiz (anger) $)_{\mathrm{acc}}\left([+\mathrm{c}]_{1},[-\mathrm{c}+\mathrm{m}]\left([-\mathrm{m}]_{2}\right)\right)$

Merge: ha-olam $[+c]$ hirgizacc et $\operatorname{Max}_{[-c+m]}$.

The world $[+c]$ angered $d_{a c c}(\operatorname{acc}) \operatorname{Max}_{[-\mathrm{c}+\mathrm{m}]}$.

cause

experiencer

(24) Reduction:
a. $\quad \underline{\mathrm{R}}_{\mathrm{c}}\left(\right.$ hirgiz) (=hitragez) $\left([-\mathrm{c}+\mathrm{m}],\left([-\mathrm{m}]_{2}\right)\right)$
(R(anger)) (experiencer, subject matter)
b. $\quad$ Merge (by (18)): $\operatorname{Max}_{[-\mathrm{c}+\mathrm{m}]}\left[\mathrm{vp}\right.$ hitragez (al ha-ma'amar $\left.\left.{ }_{[-\mathrm{m}]}\right)\right]$ $\operatorname{Max}_{[-\mathrm{c}+\mathrm{m}]}[\mathrm{vp}$ got-angry (about/at the-article $\left.[-\mathrm{m}])\right]$

The basic entry is (22a), where the subject matter role is optional (hence marked here with parenthesis). The output of the marking procedures is (22b): The all minus [-m] cluster is marked 2 , by $(16 \mathrm{a})$, the all plus cluster $[+\mathrm{c}]$ is marked 1 by $(16 b)$, but the mixed $[-c+m]$ is assigned no merging index. The conditions for accusative marking are met, as the cluster contains both a $[+]$ cluster $([+c])$ and a fully specified $[/-c]$ cluster $([-c+m])$. Hence $(16 c)$ marks the verb with ACC. (The experiencer cluster, thus, licenses the ACC feature, although it does not get a merging index.)

If the basic entry is selected for merging, we obtain a derivation in (23), where we ignore for the time being the optional subject matter role. By (18b), the argument realizing the cluster marked 1 must merge externally. The unmarked experiencer must then realize internally. This will enable it to check the ACC case in the subsequent derivation.

Alternatively, reduction may apply to the basic entry, as in (24a). Reduction (in Hebrew) changes the verb's morphology. The reduced verb in (24) bears reflexive morphology (hitpa'el), which is found in many instances of expletive reduction. But other morphological patterns are also available for (other) reduced verbs. By (17), reduction eliminates the ACC feature of the verb, but it does not effect the merging indices of the arguments.

Given the marking of the reduced entry, only one merging is possible at this stage: The unmarked experiencer $([-c+m])$ must merge externally, by (18a). (Since nothing blocks this external merging, it is the only permitted option.) If the [-m] argument is realized, as in (24b), it must merge internally, by (18b) (since it is marked 2).

This captures, then, the basic properties of the worry type verbs. In a language like English, which never marks reduction morphologically (also in the 
case of reflexive reduction), the two realizations of worry look identical. Nevertheless, (21a), repeated, realizes the basic entry, parallel to (22), while (21b) is the output of reduction, parallel to (24).
a. Something worries Lucie. cause experiencer
b. Lucie worries (about something) experiencer subject matter

Syntactically, the two derivations in (21) are completely independent, and neither involves any movement operation. The fact that the experiencer role of the basic entry realizes internally in (21a) and externally in (21b) poses no problem in the present system: This is allowed, since experiencer is one of the two mixed clusters which are not assigned a fixed merging index by the marking procedures of the lexicon.

This still leaves us with the anaphora problem unanswered. I will argue that, in fact, sentences like (21a) (or (23) have also another derivation, which does involve movement. But establishing this requires looking in more depth into the properties of the subject matter role.

\section{The subject-matter problem}

Pesetsky (1995) observed an intriguing puzzle posed by worry type experiencing verbs. Although there is ample evidence that these verbs select both the cause and the subject matter $\theta$-roles, as in (22), these two roles can never be realized together, as in (25).

(25) a. *The article angered Bill at the government.

b. $\quad *$ The doctor's letter worried Lucie about her health.

(26) a. The article made Bill angry at the government.

b. The doctor's letter made Lucie worry about her health.

Logically, the two roles are compatible, and the content intended in (25) can be easily expressed with different structures, as in (26). So there must be some linguistic generalization ruling (25) out. Pesetsky offers a syntactic account in terms of conditions on movement. However, this rests on a radical change in the view of syntax, assuming a dual system, where derivations are processed in parallel trees. Leaving this broader issue aside, it is notobvious to me that the problem at hand is syntactic. Let us explore how it could be handled in the feature system developed here ${ }^{7}$.

I assumed already that the subject matter role corresponds, in the present system, to the cluster [-m], but let us check now the intuition behind this decision. Although our focus here is on the formal properties of the feature clusters, they obviously also play a role in the interpretation. In Reinhart (2000) I argue that what they code are basic causal relations expressed by the verbconcept. Following Shen's (1985) study of the perception of causality, I suggested that a $/+c$ feature is associated with a participant (role) perceived as 
forming a sufficient, and not just a necessary, condition for the event. (All selected arguments can be viewed as necessary conditions for the event taking place, but only $[/+c]$ clusters are associated with participants perceived as both necessary and sufficient conditions.)

We assumed that the unary specification leaves unspecified the value of the argument with respect to the other feature. If a $[/+c]$ cluster is unspecified with respect to the / $\mathrm{m}$ feature, it is left open whether the 'mental state' condition also holds. Hence the verb is consistent with either a cause or an agent interpretation of the relevant argument. What is specified in a unary cluster is just the feature that defines the cluster, namely must be assumed in all interpretative realizations of the cluster.

Let us look now at the relation a subject matter role bears to the event represented e.g. in Lucie worries about the state her health. An obvious feature her health has is /-m (the mental state of a subject matter participant is never relevant). But what is its /c status? Although this role is distinct from cause, it is still possible to view Lucie's health as a cause for her worrying. Having some state of health is a necessary (enabling) condition for worrying about it. But it can also be a sufficient condition - the direct cause for worry. More broadly, in our perception of the world it is possible that the subject matter of emotion is itself the cause of this emotion. Whether it is or not for a given situation depends just on whether there is another condition we perceive as causing it. In feature terms, this means that the subject matter role is only specified as /-m, namely, it is $[-\mathrm{m}]$.

In feature terms, then, the descriptive generalization suggested by (25) is that a $[+\mathrm{c}]$ and a $[-\mathrm{m}]$ cluster cannot be both realized in a given derivation.

Once $[-\mathrm{m}]$ is recognized as a cluster defining a set of roles, we may expect to find other instantiations of this cluster, namely other roleinterpretations for it. Locative source is one. Doron (1999) noted that the same pattern we observed with worry-type experiencing verbs is also found with Hebrew verbs selecting a locative source. An example is verbs of providing nutrition or living: kiyem (maintain/sustain), pirnes (provide/support), hezin (nurture).

a. ha-ikar pirnes et mishpax-to.

The-farmer supported (acc) his-family

b. ha-mishpaxa hitparnesa me-ha-sade.

The-family supported [itself] from-the-field (made its living of the field).

c. *ha-ikar pirnes et mishpax-to me-ha-sade.

*The-farmer supported (acc) his-family from/of-the-field

Under the present analysis (which differs from Doron's), the morphology marks the verbs in (27b) as the reduced (unaccusative) form of (27a). This reduced form takes a locative-source complement. This means that this role must be part of the $\theta$-specification of the underlying verb (in (27a)). But still this argument cannot occur in the non-reduced form, as witnessed in $(27 \mathrm{c})$. So the 
pattern is precisely the same we observed with worry (in (25), though no subject matter role is involved. (Other verbs with this pattern, listed by Doron, are hishir (shed leaves), hizil (drip), hidif (emanate).)

Doron notes that the locative-source is interpretable similarly to a cause. This is, again, analogous to what we saw with worry. It means that this role is not specified for $/ \mathrm{c}$, and whether it is viewed as a cause or not, depends on whether another $[/+\mathrm{c}]$ role is realized. So this is another instance of a $[-\mathrm{m}]$ role $^{8}$. The verbal entry under consideration, then, is (28a).

a. pirnes/hezin (provide/nourish) $([+\mathrm{c}],[-\mathrm{c}-\mathrm{m}],[-\mathrm{m}])$

(cause, patient, source)

b. pirnes/hezin acc $\left([+\mathrm{c}]_{1},[-\mathrm{c}-\mathrm{m}]_{2},[-\mathrm{m}]_{2}\right)$

(cause, patient, source)

The marking procedures apply as in (28b). Unlike the experiencer cases, the second cluster of the verb is marked as obligatorily internal, which entails that the derivation based on the reduced verb, in (27b) is unaccusative, with the subject originating internally. But the pattern of realization of the [-m] cluster is the same here as with the worry type verbs: Since the external role of these entries is $[+c]$, the descriptive generalization we observed prohibits the realization of the $[-\mathrm{m}]$ role in $(27 \mathrm{c})$. But when the $[+\mathrm{c}]$ role is reduced, as in (27b), it is allowed to be realized.

A remaining question is why the $[+\mathrm{c}]$ and the $[-\mathrm{m}]$ role cannot be realized together. In Reinhart (2000) I proposed that this may reflect a broader restriction on the realization of feature clusters. A generalization which is largely assumed is that the same $\theta$-role cannot be realized twice (i.e. a verb cannot realize two identical clusters). Kremers (1998) proposes to restate this generalization as the distinctness requirement in (29a).

Cluster distinctness:

a. Two indistinct $\theta$-clusters cannot be both realized on the same predicate.

b. Distinctness: Two feature-clusters $\alpha, \beta$, are distinct iff a. they share at least one feature, and $b$. there is at least one feature or value which they do not share.

The option that (29a) opens is that a verb selects several (non identical) clusters, but nevertheless, they cannot all be realized in one derivation, because they are not sufficiently distinct. The question, then, is what counts as distinct $\theta$-clusters. I propose the definition in (29b) (which differs from the definition in Reinhart (2000)). The intuition behind (29b) is that identifying distinctness requires some shared basis for comparison. An entailment of $(29 b)$ is that indistinct clusters which are not identical can be found only with pairs of two unary clusters: When at least one cluster of a given pair is fully specified, the two clusters always have some shared feature - $/ \mathrm{c}, / \mathrm{m}$, or both - which is the basis for comparison. Hence, the only way in which they can turn indistinct is by clause (b) of (29b), namely they are identical. (E.g. $[+c+m]$ shares with $[-m]$ the feature $/ \mathrm{m}$, and they differ in all the rest. It shares with $[+\mathrm{m}]$ the feature and value $/+\mathrm{m}$, 
and they differ in the rest, etc.) With Unary features this is not so: A unary [/c] cluster never shares any feature with a unary $[/ \mathrm{m}]$ cluster, so they are always indistinct by clause (a) of (29b). The only unary clusters that can be realized together are, then, $<[+c],[-c]>$ and $<[+m],[-m]>$.

It follows from (29) then, that if a verb selects both a $[+\mathrm{c}]$ and a $[-\mathrm{m}]$ argument, as argued for the worry and the nourish types of verbs, these clusters are indistinct and hence, cannot be both realized in the same predicate, as we saw in (25b) and (27c).

Further illustration for (29), involving the [+c] cluster, is given in a footnote $^{9}$. Other clusters realizations prohibited by $(29)$ are $<[-\mathrm{c}][-\mathrm{m}]>,<[+\mathrm{m}]$ $[-\mathrm{c}]>$ and $\langle[+\mathrm{m}][+\mathrm{c}]>$. The last pair is also prohibited independently, as the marking procedures assign the index 1 to both clusters, but only one external argument can be realized per derivation. Some evidence supporting this outcome of (29) for the other two pairs is provided in Botwinick-Rotem (2001). Nevertheless, it may be still premature to conclude decisively that (29) is indeed relevant for all unary clusters. What has been clearly established is that the distinctness requirement holds for the $[+c]$ cluster, namely the generalization in (30).

(30) A [+c] cluster cannot be realized with indistinct clusters (as defined in (29b).

In section 7, I will return to further substantiation of this generalization.

\section{The anaphora pattern}

The verbal pattern in (27) includes one more member, which we have not yet considered.

(31) ha-sade kiyem/pirnes et ha-mishpaxa.

The-field ${ }_{[-m]}$ supported/sustained acc-the-family[-c-m].

(32) ha-ec hishir et al-av.

The-tree shed (acc)its-leaves.

The verb morphology in (31) is the same as in the causative (transitive) form of $(27 \mathrm{a}, \mathrm{c})$. This, in our terms, means that no reduction took place. Still, the argument that surfaces in external position is the internal [-m] role. This is even clearer in (32) (from Doron 1999).

Upon closer examination, this pattern is found also with experiencing verbs selecting the $[-\mathrm{m}]$ role. So far we assumed that in object-experiencing derivations the subject always realizes the external $[+c]$ role of the verb, as in (21a), repeated.

(21) a. The article angered Bill.

$$
\text { cause experiencer }
$$

b. Bill was angry at/about the article. 
This was based on Pesetsky's observation that (21a) does not entail (21b), since it is possible to construe (21a) such that the article made Bill angry at something else (rather than at some properties of the article itself, which is the only construal of (21b)).

Nevertheless, it is also possible to construe (21a) as equivalent to (21b), namely that Bill got angry about some properties of the article itself. The sentence, then, has two semantic construals, depending on whether the article is viewed as the cause $([+\mathrm{c}])$ or as the subject matter $([-\mathrm{m}])$. Contextual considerations may enforce disambiguation of the two construals. E.g. in (33a) it is easiest to interpret Lucie's health as the subject matter of her worry, while in (33) the cause construal is more natural, namely that the doctor's letter made Lucie worry about something else.
a. Her health worried Lucie. subject matter[-m]
b. The doctor's letter worried Lucie. cause $[+c]$

This already suggests that experiencing verbs allow also a derivation more on a par with (31-32), namely that in (33) her health realizes the internal [-m] argument. But there are also more robust indications in this direction, which brings us back to the question of anaphora:

It is widely believed that backwards bound anaphora, as in (34a), is always found with object-experiencing derivations. But, in fact, it is worse in (34b) than in (34a).
a. $\quad$ His $_{\mathrm{i}}$ health worried every patient $\mathrm{i}_{\mathrm{i}}$
b. $\quad$ ??Hisi doctor's letter worried every patient ${ }_{\mathrm{i}}$.

The contrast in (34) is not fully decisive, because it is not impossible to construe (34b) with the subject (his doctor's letter) as the subject matter of the patients' worry. The point is, however, that anaphora is enabled only if the subject is construed as the $[-\mathrm{m}]$ argument.

In section 7, we will see more direct evidence for this anaphora pattern. But for here, we may note that it is further confirmed by the entailment patterns. We saw that in (21), repeated again, the object-experiencing (21a) does not entail the subject experiencing (21b) (because the article is construed with two different roles in the two sentences). But this does not hold when the relevant anaphoric dependence is involved, as in (35): (35a) does entail (35b).

a. The article angered Bill ---->

$$
\text { cause experiencer }
$$

b. Bill was angry at/about the article.
experiencer
subject matter

a. His $\quad$ Hrade angered every student $_{i}---->$
subject matter
experiencer 
b. Every student was angry about hisi grade. experiencer subject matter

This is so, since anaphora in (35a) is permitted only if his grade is construed as the subject matter. In this case, the thematic composition is identical in (35a) and $(35 b)$, so the sentences are equivalent.

Note now that the same generalization is witnessed also with the provide type verbs:

a. [ha-sandwich shelo $\left.]_{i}\right]_{-m]}$ hezin kol yeled $\mathrm{d}_{\mathrm{i}}$ e bemeshex shavu'a [Hisi sandwich $]_{[-m]}$ nourished e every child $d_{i}$ for a week. $\begin{array}{ll}\text { source }_{1} & \left(\mathrm{t}_{1}\right) \text { patient }\end{array}$

b. ima shelo $]_{[+c]}$ hezina kol yeled $d_{\mathrm{i}}$ bemeshex shavu'a */?[Hisi mother] $]_{[+c]}$ nourished every child for a week. cause patient

With these verbs, it is easier to distinguish between the [+c] and the [-m] (source) roles, and only (36a) is readily interpreted with the subject as the source. Indeed, anaphora is much easier in (36a) than in (36b). (Note that (36b) is an instance of 'weak-crossover' which is usually weak, namely not that bad in all contexts.)

In the present system the [-m] role must merge internally (by the merging generalization (18b)). So the fact that it can also surface externally suggests that movement takes place in these derivations, namely that an analysis along the lines of Belletti and Rizzi (1988) must be available for worry type verbs (in their object-experiencer realization). This would also capture, then, the anaphora facts, which, as mentioned, were the strongest argument for their analysis.

As we saw in section 1, Belletti and Rizzi's unaccusative analysis could not work for the standard worry derivations, where the cause role is realized as subject, because these derivations do not show any unaccusative properties. (E.g. they allow passive.) But here we are considering only derivations realizing the subject matter role externally, namely a subset of the object-experiencing derivations.

\section{A movement derivation}

In fact, the present system entails that another derivation of experiencing verbs should be possible. So far we observed, in section 3 , one derivation based on the basic entry, and one based on the reduced entry. But the basic entry allows two derivations.

Let me illustrate this, again, with Hebrew, where the morphology indicates whether reduction took place. (22), repeated, is the basic entry for anger. The verb selects both a $[+\mathrm{c}]$ and a $[-\mathrm{m}]$ clusters, but the feature generalization (29) (or 30) determines that only one of them can be realized. So far we assumed that $[+c]$ is the one that realizes, and, thus, we obtained the derivation in (26), where the $[+c]$ argument merges externally. 
However, no principle dictates that it must be the $[+c]$ cluster that realizes. Let us now check what happens if we choose to realize the $[+\mathrm{m}]$ cluster instead. Assuming some V-shell structure (without entering its details), the derivation proceeds as in (37). For convenience, the merging instructions of (18) are repeated below.

$$
\begin{aligned}
& \text { a. [v } \left.\text { angered }_{\mathrm{acc}} \text { the } \operatorname{article}_{[-\mathrm{m}]}\right] \\
& \text { b. } \quad\left[v \operatorname{Max}_{[-c+m]}\left[v \text { angered }_{\mathrm{acc}} \text { the } \operatorname{article}_{[-\mathrm{m}]}\right]\right. \\
& \text { c. [v angered }{ }_{a c c}\left[\operatorname{vax}_{[-c+m]}\left[v t_{\text {angered }} \text { the article }[-m]\right]\right. \\
& \text { d. [ip the } \left.\operatorname{article}_{[-m]}\left[v p \operatorname{angered}_{a c c}\left[v \operatorname{Max}_{[-c+m]}\left[v t_{\text {angered }} t_{\text {article }}\right]\right]\right]\right]
\end{aligned}
$$

\section{CS merging instructions.}

a. When nothing rules this out, merge externally.

b. An argument realizing a cluster marked 2 merges internally; An argument with a cluster marked 1 merges externally.

The [-m] argument, marked 2, must merge internally, by (18b), which means here that it merges first, as in (37a). The experiencer argument has no merging index. In principle, it could merge externally. However, the derivation will then crash, as there will be no DP to check the ACC case-feature. (Recall that in the present system only fully specified clusters can check the ACC case.) (18a) would force this argument to merge externally only if nothing rules this out, but case considerations rule this option out in this derivation. So the argument can merge as in (37b) (or the derivation crashes. As noted in the discussion of (18), (18a) has an economy flavor.) The verb then moves in (37c), as is standard in Vshell analyses. In this configuration Max can check the ACC case of the verb. Next, the [-m] argument (the article) moves to satisfy the EPP, as in (37d).

As further evidence that derivations like (37) must exist for worry-type experiencing verbs, note that when the [-m] role is realized by a clause, these verbs allow expletive subjects, as in (38). The same is found in Hebrew, with an empty expletive, as in (39).

\section{It angered/surprised/scared/excited him [that he failed $]_{[-\mathrm{m}]}$.}

0 hirgiz et Max [she+hu nixshal]

[It] angered (acc) Max [that he failed].

These little studied derivations appear to pose a serious problem to Burzio's generalization: The verb clearly assigns the accusative case, and still no external role is realized. As noted in section 1, under Belletti and Rizzi's (1988) analysis, the derivation of experiencing verbs is indeed unaccusative, so the expletive is 
not a surprising option. But, unlike some other cases in Italian, it is obvious that the verb here does assign accusatives, so this remains a violation of Burzio's generalization, and a mystery.

In the present system, no problem arises here. The accusative feature is licensed at the full basic entry, where a $[+c]$ (external) role is present. Since no reduction operation applied, this feature stays and needs to be checked, regardless of whether $[+\mathrm{c}]$ or $[-\mathrm{m}]$ are selected for realization. If the $[-\mathrm{m}]$ cluster is the one realized, the VP phase of (38) parallels that of (37c), as in (40a).

$$
\begin{aligned}
& \text { a. } \quad\left[\mathrm { vp } \text { angered } { } _ { \mathrm { acc } } \left[\mathrm{v} \operatorname{him}_{[-\mathrm{c}+\mathrm{m}]}\left[\mathrm{v} \mathrm{t}_{\text {angered }}[\text { that he won }]_{[-\mathrm{m}]}\right]\right.\right. \\
& \text { b. } \quad\left[\text { ip it } \left[\mathrm{vp} \text { angered }{ }_{\text {acc }}[\mathrm{v} \text { him }[-\mathrm{c}+\mathrm{m}]\right.\right. \\
& {[\mathrm{v} \text { tangered }}
\end{aligned}
$$

At this stage, either the embedded [-m] clause moves, or an expletive is merged to satisfy the EPP, as in (40b) $)^{10}$.

The derivations under consideration here, then, are not strictly unaccusative, as the accusative case is present. Nevertheless, they share properties with unaccusative derivations - the movement of an internal argument. Though I cannot elaborate on this here, the arguments we surveyed in section 1 against Belletti and Rizzi's analysis (such as the availability of passive) hold only for derivations realizing the $[+\mathrm{c}]$ role, and not for those where the argument is clearly [-m].

The anaphora facts now follow clearly: Bound anaphora is possible in the movement derivation, as in (41a). As seen in greater detail in (41b), the pronoun copy (trace) is c-commanded by the quantified antecedent, so at this stage, the c-command requirement on bound anaphora is met.

a. $\quad\left[\text { His }_{\mathrm{i}} \text { health }\right]_{j[-\mathrm{m}]}$ worried every patient $\mathrm{i}_{\mathrm{j}}$

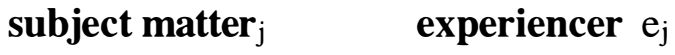

b. [ip his health
health] $]\left[\mathrm{vp}\right.$ worried ${ }_{\text {acc }}[\mathrm{v}$ every patient $[-\mathrm{c}+\mathrm{m}]$

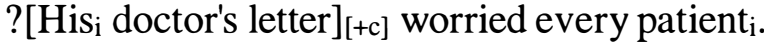 cause experiencer}

But, as observed in section 5 , this is only possible if the subject is construed as the subject matter. This follows now, since only if the subject is the [-m] argument the derivation involves movement. If the subject is construed as the cause, as in (42), it merges directly externally, and at no stage is the c-command requirement met.

The movement analysis is available, as we saw, only for the [-m] argument of a worry type verb. For English, it appears that most verbs which select both a $[+\mathrm{c}]$ and a $[-\mathrm{m}]$ cluster can equally realize any of them. But Friedemann (2000) notes that there are verbs which allow only the [-m] realization, e.g. fascinate. I assume that these verbs originate nevertheless as standard worry verbs, but the realization option is fixed (frozen) in the lexicon. (In the present system, this explains why the accusative case is still realized in the derivations.) Friedemann points out that there are many such verbs in French. E.g. worry has two entries: inquieter, which behaves like the English worry, and 
preoccuper, which allows only $[-\mathrm{m}]$ realization.

\section{Minimal pairs}

The movement experiencing-derivation rests crucially on the conflict of the $[+c]$ and $[-\mathrm{m}]$ clusters, which enables suppression of the $[+\mathrm{c}]$ role. We may observe now that not all experiencing verbs select precisely these clusters, and that a minimal difference in the cluster selection of verbs entails a substantial difference in the set of syntactic derivations which they permit.

It was noted by Pesetsky (1995) that some experiencing verbs do allow all their three $\theta$-roles to be realized in their non reduced form (objectexperiencing), and, in fact, some even require this. This is illustrated in the (i) sentences of (43).
a. i. The press biased the judge (against the defendant).
ii. The judge was biased against the defendant.
b. i. This alienated her from her colleagues.
ii. She was alienated from her colleagues.
c. i. ze hirgil oto le-oni. (This accustomed him to poverty.) ii. hu hitragel le-oni. (He accustomed to poverty.)

If the external $[+c]$ role is reduced, the result is the two place (subjectexperiencing) entry, exemplified in the (ii) sentences. As we noted for the other experiencing verbs, in English, the reduced form is hardly available, and a passive form is used instead. But in Hebrew these verbs do have a reduced form. In (43c), we see that the reduced form bears the morphological marks of reduction, as with the other experiencing (and unaccusative) verbs.

Other examples of such three-place verbs, listed by Pesetsky (p.216), are: arouse, incline, provoke, stimulate, estrange, habituate. In Pesetsky's framework, these pose a problem which necessitates a certain amount of stipulations. In the features approach, we should search the answer in the properties of the $\theta$-roles of these entries.

Unlike the worry verbs, the third argument in the (i) entries cannot be naturally construed as a potential cause (sufficient condition) of the reported state of mind. This can be checked by comparing the pair in (21), repeated again, to (44).

(21) a. The article angered Bill. cause - $[+c]$

b. Bill was angry at/about the article.

\section{subject matter-[-m]}

(44) a. The press biased the judge. cause - $[+\mathbf{c}]$

b. The judge was biased against the press. 
The (a) sentence in both is derived from the basic entry, and the (b) sentence from the reduced one. In (21), it took some effort to prove that the sentences are not equivalent, while in (44) the option does not even arise, as they are so clearly different. The reason (21) requires thought is that the roles the article bears in the two derivations are potentially related, and are, thus, hard to distinguish. But in (44), they are clearly distinct: the press in (44b) cannot be construed as a potential cause, which means it must have the /-c feature.

Intuitively, the third (?) role in the bias type verbs resembles more the goal role (or perhaps Pesetsky's target), than the subject matter goal. In the present system, goal is [-c], and as we diagnosed already the ? cluster as containing a /-c feature, I assume it is, like goal, a [-c] cluster ${ }^{1}$.

The basic entry for bias verbs is, then, given in (45), which includes already the output of the marking procedures.

$$
\text { bias }_{\mathrm{acc}}\left([+\mathrm{c}]_{1},[-\mathrm{c}+\mathrm{m}],[-\mathrm{c}]_{2}\right) \text { (cause, experiencer, goal/target) }
$$$$
\text { worry }_{\mathrm{acc}}\left([+\mathrm{c}]_{1},[-\mathrm{c}+\mathrm{m}],[-\mathrm{m}]_{2}\right) \text { (cause, experiencer, subject matter) }
$$

The bias verbs, thus, differ minimally from the worry verbs in (46): They differ only in whether the third argument is [-m] or [-c]. However, this minimal difference entails substantial differences in the syntactic realizations the verb permits.

First, this difference explains the realization problem we started with (in (43)). Recall the cluster distinctness definition in (29), repeated.

\section{Cluster distinctness:}

a. Two indistinct $\theta$-clusters cannot be both realized on the same predicate.

b. Distinctness: Two feature-clusters $\alpha, \beta$, are distinct iff a. they share at least one feature, and $b$. there is at least one feature or value which they do not share.

By $(29 b)$ the cluster $[+c]$ is indistinct from $[-m]$, but it is distinct from [-c]. Hence, (29a) (or 30) allows only two clusters of worry to realize in a given derivation. But for the bias verbs, it allows all three clusters to realize together, which, as we have just observed, is indeed the case.

This has further implications: As we saw in section 6, in the worry verbs, the realization of the $[+c]$ role is optional. Hence, the verbs allow a movement derivation, based on realizing only the [-m] role. However, this option is licensed only by (29a): since the verbs select the indistinct pair $<[+c],[-$ $\mathrm{m}]>$, one of them is not realized. Nothing else in the system permits arbitrary non-realization of the $[+c]$ cluster $^{12}$. Hence, this option is not available for the bias verbs.

The first implication of this difference regards expletive subjects. As we saw, the worry verbs allow derivations like (47a), based on realizing the [-m] cluster. Since bias verbs do not allow such realization, we expect them not to allow an expletive subject, which is indeed witnessed in (47b). 
Expletives:

(47) a. It worried/surprised/scared Max that he was always winning.

b. *It biased/alienated/habituated/estranged/inclined Max [that he was always winning] / [to always win].

Next, we saw that the derivation based on realizing only the $[-\mathrm{m}]$ cluster is what enables backward anaphora in worry verbs, as in (48).

anaphora:

(48) Hisi health worried every patient $t_{i}$

(49) a. $\quad * /$ ? His $\mathrm{H}_{\mathrm{i}}$ upbringing biased every juror $\mathrm{i}_{\mathrm{i}}$ (against the defendant).

b. $\quad$ ?/*His $\mathrm{H}_{\mathrm{i}}$ musical taste alienated every pianist $\mathrm{i}_{\mathrm{i}}$ from the audience.

Since the bias verbs do not have such derivation, nothing licenses this type of anaphora. Indeed the sentences of (49) are just standard instances of weak crossover, namely, they are worse than (48).

\section{Endnotes}

* Earlier versions of this lecture were given in IATL, Tel Aviv, June 2000, and in the Leiden colloquium, September 2000. Along with the audience of these lectures, I wish to thank Marc- Ariel Friedemann, Idan Landau and Tali Siloni for extensive comments and discussion.

${ }^{1}$ This may seem puzzling, given that in (1a) the verb seems to assign the accusative case (Max worried her). But Belletti and Rizzi, who focused their analysis on Italian, argued that at least in the Italian cases, this is not a real accusative.

${ }^{2}$ Pesetsky, in fact, argues that worry type verbs are listed as unaccusative (oneplace) verbs: The object-experiencer derivation (7b) reflects, thus, the basic verb entry, and the subject originates internally. (7a) is derived by a causativization operation from (7b). But these aspects of his analysis are debatable. In Reinhart (2000) I argue, first, that derivations like (7b) show unergative rather than unaccusative properties, and, next, that the familiar causativisation operation cannot derive (7b) from (7a).

${ }^{3}$ Candidates for this feature-cluster are the external arguments of verbs like know or love. Also the semi-agent argument of verbs like laugh, cry, sleep, ( as well as sit and stand in their unergative entry). These verbs require an animate argument, but do not involve necessarily agency or a causal relation with this argument.

${ }^{4}$ In Reinhart (2000), I assumed that the role cause is the cluster [+c-m], i.e. that instrument and cause realize the same feature cluster, and the relevant interpretation is determined contextually. [+c] was assumed to be a special feature cluster which is consistent with the three roles (agent, cause, instrument). However, I did not find subsequently any verb which selects an argument which is obligatorily only a cause (i.e. cannot be realized also by an 
instrument or an agent). Hence, this assumption is not justified.

${ }^{5}$ The varying interpretation of the external role is found only with $[+c]$ verbs. Verbs selecting an agent $([+\mathrm{c}+\mathrm{m}])$ have fixed interpretation, as illustrated in (i).

$$
\begin{aligned}
& V([+c+m],[-c+m] \\
& \begin{array}{l}
\text { a. The baby/ } / \text { the spoon } / * \text { hunger ate the soup. } \\
\text { b. Lucie/ } * \text { The razor } / * \text { the heat shaved Max. } \\
\text { c. Lucie/ *the snow } / \text { the desire to feel warm dressed Max }
\end{array}
\end{aligned}
$$

Levin and Rappaport (1995) noted a set of 'manner' verbs which allow the subject to be interpreted as either an agent or an instrument (but not a cause), as in (ii).

(ii) Max/the knife $/ *$ the heat of the oven peeled the tomato.

I discuss these in Reinhart (2001). (The basic analysis is that these select an agent and an obligatory instrument. Since both have the feature $/+c$ only one of them is obligatory, and the mapping system we turn to below determines that the one realized merges externally.

${ }^{6}$ In Reinhart (2000) I assumed that it is a free operation, applying to any external role, but it is subject to a special condition that $[/+\mathrm{m}]$ roles cannot be reduced. However in practice, this operation only applies to $[+c]$ arguments. Hence we may as well skip the special condition and build this fact into the definition of the operation, in (25). There are also empirical reasons for this change, mentioned briefly in Reinhart (2001).

${ }^{7}$ Pesetsky mentions briefly the option of capturing this problem with feature restrictions (footnote 60), and dismisses it on grounds which are irrelevant in the present framework.

${ }^{8}$ Given the two instances of a [-m] role we observed, a plausible hypothesis is that a role with this feature is interpreted as 'subject matter' with verbs selecting a $[+\mathrm{m}]$ complement (experiencer), and as a source otherwise.

${ }^{9}$ As further illustration of this generalization, note that there is a subset of both the worry and the provide type verbs which has peculiar properties: Verbs like interest and convince appear to allow their [-m] cluster to realize in the nonreduced verbal form, as in (ib), which contrasts with (25b), repeated.

(25) b. *The doctor worried Lucie about her health.

(i) a. Lucie/the article interested Max.

b. Lucie interested Max in linguistics.

c. $\quad * /$ ? The article interested Max in linguistics.

But this is only possible when the external argument is animate - (ic) is much worse. The same is found with the Hebrew verb kiyem (sustain), which in all other respects belongs to the provide group. Again, (iia) is much better than either (iib,c) or (27c), repeated. 
c. *ha-ikar pirnes et mishpaxt-o me-ha-sade.

*The-farmer supported (acc)-his-family from/of-the-field

(ii) a. ha-ikar kiyem et mishpaxt-o me-ha-sade.

The-farmer sustained (acc)-his-family from/of-the-field.

b. *ha-sade kiyem et ha-mishpaxa me-ha-tiras.

*The-field sustained (acc) the-family from/of the corn.

c. *ha-xisaxon kiyem et ha-mishpaxa me-ha-ribit.

*The saving (account) sustained the family of the interest.

This would follow from (29), if we assume that interest and kiyem-sustain select, along with their $[+\mathrm{c}]$ cluster also a $[+\mathrm{m}]$ cluster. (Generally, the $[+\mathrm{m}]$ cluster, which is unspecified for /c, allows, but does not force, a volitional or 'agentive' interpretation. In all other respects, the analysis will work just the same if the added cluster is an agent $-[+\mathrm{c}+\mathrm{m}]$, rather than $[+\mathrm{m}]$.) The verbs' entries are then, as in (iii).

(iii) a. interest $\mathrm{acc}_{\mathrm{ac}}\left([+\mathrm{c}]_{1},[+\mathrm{m}]_{1},[-\mathrm{c}+\mathrm{m}]_{2},[-\mathrm{m}]_{2}\right)$

b. kiyem (sustain) $)_{\text {acc }}\left([+\mathrm{c}]_{1},[+\mathrm{m}]_{1},[-\mathrm{c}-\mathrm{m}]_{2},[-\mathrm{m}]_{2}\right)$

The $[+\mathrm{c}]$ and the $[+\mathrm{m}]$ clusters can never realize together: They are indistinct by (29b), but independently, as [+] clusters, they are both assigned the index 1, and only one argument can realize externally. So the actual derivation selects only one of these clusters for realization. If the $[+c]$ argument is selected, we get the same pattern as with the standard worry or provide verbs, namely, the $[+\mathrm{c}]$ and the [-m] clusters cannot be both realized. But the [+m] cluster is defined as distinct from the [-m] cluster (as they share a feature and differ in its value). So if this cluster is selected for the derivation, the $[-\mathrm{m}]$ cluster can be realized as well. The $[+\mathrm{m}]$ cluster, however, can only be realized with an animate DP. (This is a general restriction found with all $[/+\mathrm{m}]$ clusters, like agent and experiencer.) So, only in (ib) and (iia) it is possible to construe the subject as realizing the $[+\mathrm{m}]$ cluster.

${ }^{10} \mathrm{An}$ independent question, the answer to which I do not know, is why this expletive is only possible if the complement is a clause.

${ }^{11}[-c]$ clusters allow in some contexts a /+m interpretation (see Reinhart 2001). But this depends on the verb, and is not the case with the present role. In the cases allowing this, like escape. the verb concept has mental properties, but no experiencing cluster.

${ }^{12}$ There is, in fact, another condition which allows a $[+c]$ cluster not to be realized (discussed in Reinhart (2001)): When a verb selects two [/+c] clusters, one of them is optionally not realized: This holds for $[+\mathrm{c}],[+\mathrm{c}+\mathrm{m}]$ and $[+\mathrm{c}-\mathrm{m}]$. Verbs which select both a [+c-m] cluster (roughly instrument) and either a $[+\mathrm{c}]$ or a $[+\mathrm{c}+\mathrm{m}]$ arguments are, most notably, 'manner verbs'. In such cases it is possible to realize only the instrument. However, this role will realize externally, given the mapping conditions above. 


\section{References}

Belletti, A., and L. Rizzi (1988) "Psych-verbs and $\theta$ theory", Natural Language and Linguistic Theory 6, 291-352.

Botwinick-Rotem, Irena (2001), "The phenomenon of obligatory PPs", ms. Tel Aviv University.

Doron, Edit, (1999), "Agency and voice: The semantics of the Semitic Templates", Manuscript, The Hebrew University, Jerusalem.

Friedemann, Marc Ariel (2000), "Experiencing verbs in French", lecture in the Lexicon Workshop, Utrecht, October 2000.

Kremers, Joost. (1999) "Theta role mapping", ms., University of Nijmegen.

Pesetsky, David, (1995) Zero Syntax: Experiencers and Cascades, MIT Press, Cambridge, Mass.

Reinhart, Tanya (2000), The Theta System: Syntactic realization of verbal concepts, OTS Working papers, 00.0/TL (available also at http://www.let.uu.nl/ tanya.reinhart)

Reinhart, Tanya (2001), "A synopsis of the Theta System", ms. available at http://www.tau.ac.il/ reinhart (WP51),

or http://www.let.uu.nl/ tanya.reinhart (Word).

Shen, Yeshayahu, (1985) The Structure of Action in the Short Narrative Text, Unpublished Ph.D Dissertation, Tel Aviv University. 